\title{
Spin wave effects in transport between a ferromagnet and a Weyl semimetal surface
}

\author{
A. Kononov, ${ }^{1}$ O.O. Shvetsov, ${ }^{1,2}$ A.V. Timonina, ${ }^{1}$ N.N. Kolesnikov, ${ }^{1}$ and E.V. Deviatov ${ }^{1}$ \\ ${ }^{1}$ Institute of Solid State Physics of the Russian Academy of Sciences, \\ Chernogolovka, Moscow District, 2 Academician Ossipyan str., 142432 Russia \\ ${ }^{2}$ Moscow Institute of Physics and Technology, Institutsky per. 9, Dolgoprudny, 141700 Russia
}

(Dated: July 11, 2018)

\begin{abstract}
We experimentally investigate spin-polarized transport between a ferromagnetic Ni electrode and a surface of Weyl semimetal, realized in a thick $\mathrm{WTe}_{2}$ single crystal. For highly-transparent Ni-WTe $\mathrm{W}_{2}$ planar junctions, we observe non-Ohmic $d V / d I(I)$ behavior with an overall increase of differential resistance $d V / d I$ with current bias, which is accomplished by current-induced switchings. This behavior is inconsistent with trivial interface scattering, but it is well known for spin-polarized transport with magnon emission. Thus, we interpret the experimental results in terms of spin wave excitation in spin textures in the $\mathrm{WTe}_{2}$ topological surface states, which is supported by the obtained magnetic field and temperature $d V / d I(I)$ dependencies.
\end{abstract}

PACS numbers: $73.40 . \mathrm{Qv} 71.30 .+\mathrm{h}$

A strong area of interest in condensed matter physics is topological materials [1 -4], which combines many nontrivial effects, table top test ground for high-energy physics theories and huge potential for applications, for example in spintronics or quantum computing. Recently new classes of topological materials with gapless bulk spectra called Dirac and Weyl semimetals have been proposed [5]. Similarly to topological insulators, Weyl semimetals have topologically protected Fermi arc surface states, which are connecting projections of Weyl nodes on the surface Brillouin zone [5].

$\mathrm{WTe}_{2}$ is one of the realizations of type-II Weyl semimetal [6], where energy spectrum is tilted in momentum-energy space [7]. $\mathrm{WTe}_{2}$ demonstrates giant nonsaturating magnetoresistance [8, 9]. Now it is connected with complex spin textures in $\mathrm{WTe}_{2}$ [10 12]. Spin- and angle- resolved photoemission spectroscopy (SARPES) data indeed demonstrate spin-polarized surface Fermi arcs, and spin polarized Fermi pockets in bulk spectrum [13, 14], see Fig. 1]

Intriguing spin properties of Weyl semimetals make it attractive material for spin investigations. Giant intrinsic Spin Hall Effect was recently predicted in TaAs based Weyl semimetals [15] while SARPES measurements demonstrated nearly full spin polarization of Fermi arcs in TaAs [16, 17]. Currently there are two main spin transport approaches: illumination with polarized light and spin injection from ferromagnetic contact [18]. In the latter case one can additionally expect back action of the semimetal on the ferromagnet in the form of spin-torque, which could lead even to remagnetization of ferromagnetic contact [19]. The generation of both out-of-plane and in-plane spin-torque has been demonstrated recently in few layers $\mathrm{WTe}_{2}$ at room temperature with ST-FMR and second harmonic Hall measurements [20]. On the other hand, current-induced excitation of spin waves, or magnons, is possible at large electrical current densities for normal-ferromagnet junctions 21 24]. Thus, it is rea-

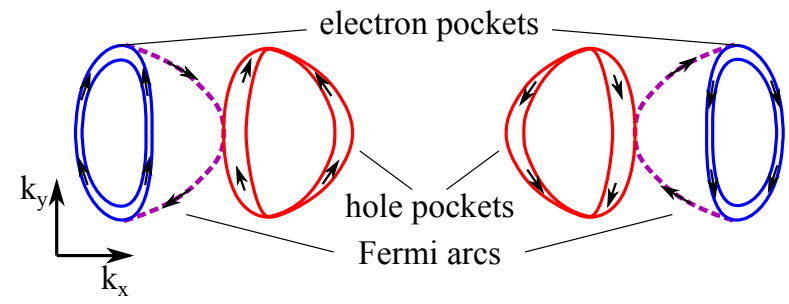

FIG. 1. (Color online) Sketch of Fermi arcs in (001) $\mathrm{WTe}_{2}$ surface Brillouin zone, and spin polarized Fermi pockets in bulk $\mathrm{WTe}_{2}$ spectrum [13, 14]. Arrows indicate spin projections, which are defined by the Weyl surface states dispersion due to spin-momentum locking 10 12].

sonable to study spin-polarized transport between a ferromagnet and a Weyl semimetal surface.

Here, we experimentally investigate spin-polarized transport between a ferromagnetic $\mathrm{Ni}$ electrode and a surface of Weyl semimetal, realized in a thick $\mathrm{WTe}_{2}$ single crystal. For highly-transparent $\mathrm{Ni}-\mathrm{WTe}_{2}$ planar junctions, we observe non-Ohmic $d V / d I(I)$ behavior with an overall increase of differential resistance $d V / d I$ with current bias, which is accomplished by current-induced switchings. This behavior is inconsistent with trivial interface scattering, but it is well known for spin-polarized transport with magnon emission. Thus, we interpret the experimental results in terms of spin wave excitation in spin textures in the $\mathrm{WTe}_{2}$ topological surface states, which is supported by the obtained magnetic field and temperature $d V / d I(I)$ dependencies.

$\mathrm{WTe}_{2}$ compound was synthesized from elements by reaction of metal with tellurium vapor in the sealed silica ampule. The $\mathrm{WTe}_{2}$ crystals were grown by the twostage iodine transport [25], that previously was successfully applied [25, 26] for growth of other metal chalcogenides like $\mathrm{NbS}_{2}$ and $\mathrm{CrNb}_{3} \mathrm{~S}_{6}$. The $\mathrm{WTe}_{2}$ composition is verified by energy-dispersive $\mathrm{X}$-ray spectroscopy. The X-ray diffraction (Oxford diffraction Gemini-A, MoK $\alpha$ ) 
a)

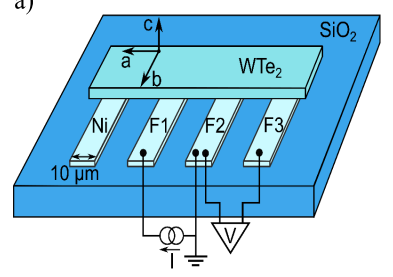

b)

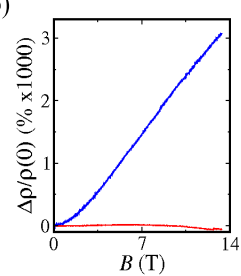

FIG. 2. (Color online) (a) Sketch of the sample with nickel contacts to the bottom surface of a $\mathrm{WTe}_{2}$ crystal (not to the scale). $50 \mathrm{~nm}$ thick ferromagnetic nickel leads are formed on the insulating $\mathrm{SiO}_{2}$ substrate. $\mathrm{A} \mathrm{WTe}_{2}$ single crystal is transferred on top of the leads with $\approx 10 \mu \mathrm{m}$ overlap, forming

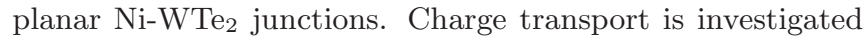
in a standard three-point technique: the studied contact (F2) is grounded and two other contacts (F1 and F3) are used for applying current and measuring $\mathrm{WTe}_{2}$ potential. The main $\mathrm{WTe}_{2}$ crystallographic directions are denoted by arrows. (b) Large positive magnetoresistance $\rho(B)-\rho(B=0) / \rho(B=0)$ for our $\mathrm{WTe}_{2}$ samples at $1.2 \mathrm{~K}$ in normal magnetic field (the blue curve). It goes to zero in parallel one (the red curve), as it has been shown for $\mathrm{WTe}_{2}$ Weyl semimetal [8]. The current is parallel to the $a$ axis of $\mathrm{WTe}_{2}$.

confirms $P m n 2_{1}$ orthorhombic single crystal $\mathrm{WTe}_{2}$ with lattice parameters $a=3.4875 \AA, b=6.2672 \AA$, and $c=14.0630 \AA$.

A sample sketch is presented in Fig. 2(a). $50 \mathrm{~nm}$ thick nickel film is thermally evaporated on the insulating $\mathrm{SiO}_{2}$ substrate mounted on the in-plane magnetized sample holder. $10 \mu \mathrm{m}$ wide ferromagnetic leads are formed by photolithography and lift-off technique. The $\mathrm{WTe}_{2}$ crystal (with dimensions $500 \mu \mathrm{m} \times 100 \mu \mathrm{m} \times 0.5 \mu \mathrm{m}$ ) is transferred on top of the leads with $\approx 10 \times 10 \mu \mathrm{m}^{2}$ overlap and weakly pressed to form planar Ni-WTe 2 junctions.

We investigate transport properties of single $\mathrm{Ni}-\mathrm{WTe}_{2}$ junction by a three-point technique, see Fig. 2 (a): a studied contact F2 is grounded, two other contacts F1 and F3 are employed to apply current and measure voltage respectively. To obtain $d V / d I(I)$ characteristics we sweep dc-current modulated by low (below $2 \mu \mathrm{A}$, $f=2 \mathrm{kHz}$ ) ac current. We measure dc and ac voltage simultaneously using voltmeter and lock-in amplifier correspondingly. Measured ac signal is independent of frequency in $1-5 \mathrm{kHz}$ range, which is defined by applied ac filters.

In a three-point technique, the measured potential $V$ reflects in-series connected resistances of the $\mathrm{Ni}^{-\mathrm{WTe}_{2}}$ junction, some part of the $\mathrm{WTe}_{2}$ crystal, and the $\mathrm{Ni}$ lead with the grounding wire. To exclude the latter term, additional connection to the grounded F2 lead is used, as depicted in Fig. 2, From $d V / d I(I)$ independence on the particular choice of current and voltage probes to the $\mathrm{WTe}_{2}$ crystal, we verify that the $\mathrm{Ni}-\mathrm{WTe}_{2}$ junction resistance dominates in the obtained $d V / d I(I)$ curves.

We check by standard magnetoresistance measurements that our $\mathrm{WTe}_{2}$ samples demonstrate large, non-

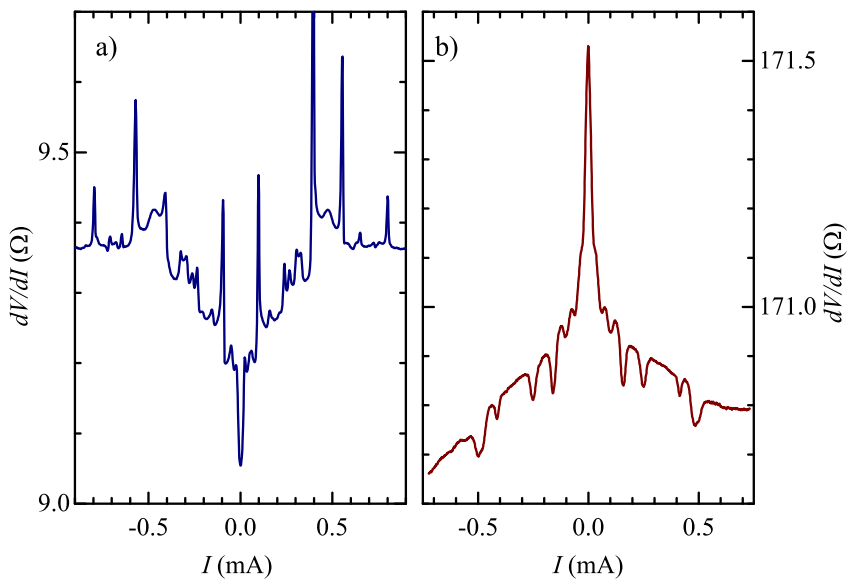

FIG. 3. (Color online) Typical examples of non-Ohmic $d V / d I(I)$ behavior for the two limiting cases of $\mathrm{Ni}_{-} \mathrm{WTe}_{2}$ junction resistance: (a) for transparent $\mathrm{Ni}-\mathrm{WTe}_{2}$ interface, $d V / d I$ is unexpectedly rising at low biases with saturation at higher ones; (b) for resistive junctions, $d V / d I$ is diminishing with bias, which is usual tunnel behavior. In both cases, currentinduced switching of $d V / d I$ can be seen as sharp $d V / d I$ peaks or dips, which are symmetric with respect to the bias sign. These features are well reproducible in different cooling cycles. The curves are obtained at $30 \mathrm{mK}$ in zero magnetic field.

saturating positive magnetoresistance $\rho(B)-\rho(B=$ $0) / \rho(B=0)$ in normal magnetic field, which goes to zero in parallel one, see Fig. 2 (b), as it has been shown for $\mathrm{WTe}_{2}$ Weyl semimetal [8]. To extract features specific to $\mathrm{WTe}_{2}$ Weyl semimetal surface states, the measurements are performed in a dilution refrigerator at temperatures from $30 \mathrm{mK}$ to $1.2 \mathrm{~K}$ with different orientations of the magnetic field to the junction plane.

Despite of equally prepared $\mathrm{Ni}-\mathrm{WTe}_{2}$ junctions, there are serious device-to-device fluctuations of the junction resistance. Fig. 3 provides typical examples of lowtemperature $d V / d I(I)$ characteristics for the two limiting cases.

For the transparent interface with low $\mathrm{Ni}^{-} \mathrm{WTe}_{2}$ junction resistance, $d V / d I$ is rising at low biases with saturation at higher ones, see Fig. 3 (a). This behavior is inconsistent with trivial impurity or roughness scattering at the interface, which can generally be described as tunneling through a potential barrier. On the other hand, an overall symmetric increase in $d V / d I$ is a familiar effect for electron scattering by emission of phonons and magnons [27].

In contrast, $d V / d I(I)$ demonstrates clear tunnel behavior for low-transparency junctions, see Fig. 3 (b): $d V / d I(I)$ is slightly asymmetric, the differential resistance $d V / d I$ is diminishing with bias.

For both realizations of $\mathrm{Ni}^{-\mathrm{WTe}_{2}}$ junctions, we observe current-induced switchings of $d V / d I$ at high currents. They appear as sharp $d V / d I$ peaks or dips in Fig. 3 (a) and (b), respectively. These $d V / d I$ features 


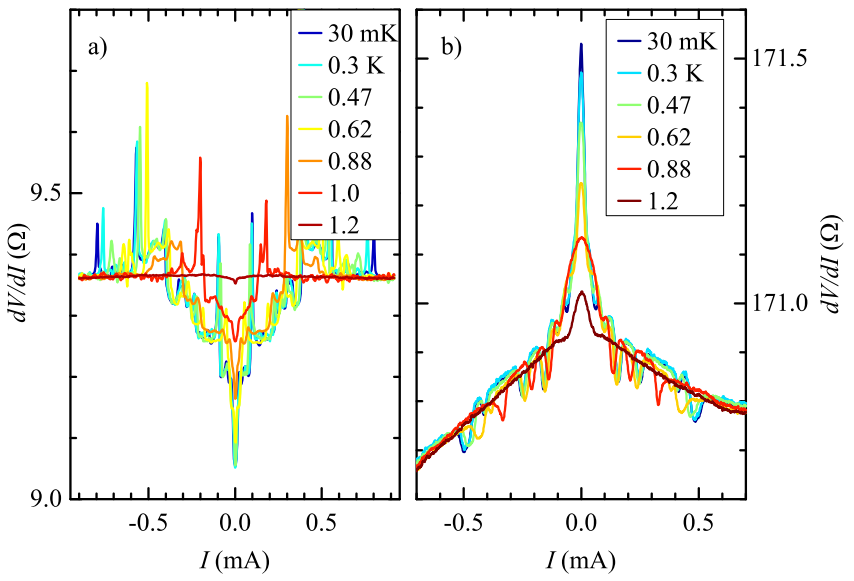

FIG. 4. (Color online) Temperature evolution of $d V / d I(I)$ characteristics for high- (a) and low- (b) transparency Ni$\mathrm{WTe}_{2}$ junctions. The effect of temperature is weak below $0.5 \mathrm{~K}$. At higher temperatures, $d V / d I$ dips and peaks amplitudes and overall $d V / d I(I)$ non-linearity are diminishing, until their complete disappearance above $1 \mathrm{~K}$. The curves are obtained in zero magnetic field.

are well reproducible in different cooling cycles. They are symmetric with respect to the current sign. There is no noticeable hysteresis with the current sweep direction for experimental $d V / d I(I)$ curves.

The observed $d V / d I(I)$ non-linearity as well as current-induced $d V / d I$ switchings are sensitive to the magnetic field and temperature.

Fig. 4 shows temperature evolution of $d V / d I(I)$ characteristics for high- and low-transparency junctions, see (a) and (b) panels, respectively. The effect of temperature is weak below $0.5 \mathrm{~K}$. At higher temperatures, $d V / d I(I)$ non-linearity is diminishing. Above $1 \mathrm{~K}$, the

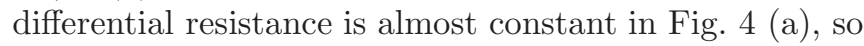
$d V / d I(I)$ s are of standard Ohmic behavior. In contrast, $d V / d I(I)$ is still non-linear for the resistive junction in Fig. (4 (b), while $d V / d I$ dips are also suppressed above $1 \mathrm{~K}$.

Fig. 5 demonstrates evolution of $d V / d I(I)$ curves with magnetic field, which is applied along a, b and c $\mathrm{WTe}_{2}$ crystal axes, respectively. The effect of magnetic field is sophisticated: in high fields, the zero-bias nonlinearity is suppressed, while the level of $d V / d I(I)$ high-current saturation is unchanged, so that $d V / d I(I)$ curve is of clear Ohmic behavior above some magnetic field. This field is smaller for normal field orientation, see Fig. 5 (c), while there is no difference for two in-plane orientations, cp. Fig. 5 (a) and (b). In lower fields, the positions of $d V / d I$ current-induced switchings are shifting to smaller currents. The effect of magnetic field on the low-transparent junction is similar to the presented in Fig. 5. The gradual evolution of switchings' positions also proves excellent reproducibility of these $d V / d I$ features in addition to their stability in thermal cycling.
We should connect the obtained results with spindependent transport between a ferromagnetic $\mathrm{Ni}$ lead and $\mathrm{WTe}_{2}$ surface states:

(i) A ferromagnetic lead is essential, since neither current-induced $d V / d I$ switchings nor an overall symmetric increase in $d V / d I$ can be observed for normal or superconducting leads to a single $\mathrm{WTe}_{2}$ crystal for different junction transparencies 28, 29].

(ii) Both current-induced $d V / d I$ switchings and overall $d V / d I(I)$ behavior can be controlled by magnetic field, see Fig. 圃,

(iii) Strong temperature dependence in the $30 \mathrm{mK}$ $1.2 \mathrm{~K}$ range can only originate from $\mathrm{WTe}_{2}$ surface state, since transport properties of Ni layer and well compensated $\mathrm{WTe}_{2}$ bulk carriers [9] are invariant in this temperature range.

Spin effects can be anticipated in $\mathrm{WTe}_{2}$ surface states due to the presence of spin textures in the $\mathrm{WTe}_{2}$ Fermi $\operatorname{arcs}$ 10, 11, 13, 14], see Fig. 1. In principle, a junction between a ferromagnetic Ni layer and a $\mathrm{WTe}_{2}$ surface can be regarded as a spin valve device. The spin valves are the sandwich structures, where spin-dependent scattering affects the magnetic moments of the spin-polarized layers, while their mutual orientation defines the differential resistance 27]. $d V / d I$ switchings have been reported for spin valves 27], but they are necessarily asymmetric with respect to the bias sign, and also accomplished by well-defined hysteresis [27], which is obviously not the case in Figs. 3 and 5 .

Inelastic transport with magnon emission 24] is a more realistic variant, since the switchings are governed [22] by magnetic field in Fig. 5.

Let us start from the low-transparent junction in Figs. 3 (b) and (b). Trivial tunneling is the main effect, which results in a standard non-linear $d V / d I(I)$ curve with $d V / d I$ diminishing with bias increase. In tunneling events, hot electrons appear above the Fermi level. They thermalize by scattering with lattice defects, phonons, or other electrons. This process is accomplished by spin polarization of the ferromagnetic lead and spin textures in the $\mathrm{WTe}_{2}$ surface state. In this case, hot electrons should additionally rotate their spins to be absorbed. Conservation of total spin results in excitation of a magnon, which opens an additional inelastic channel. Thus, the current is enhanced, which is observed as sharp dips in differential resistance $d V / d I$, as it has also been previously reported for the vacuum-separated metallic contacts [24].

Spin-wave effects are even clearer for highlytransparent junctions, see Fig. 3 (a), because of negligible interface barrier. For example, the current-induced switchings can not be connected with the potentially inhomogeneous interface in this case.

The crucial point is that the low-temperature zero-bias resistance is smaller than the value, obtained at high bi- 


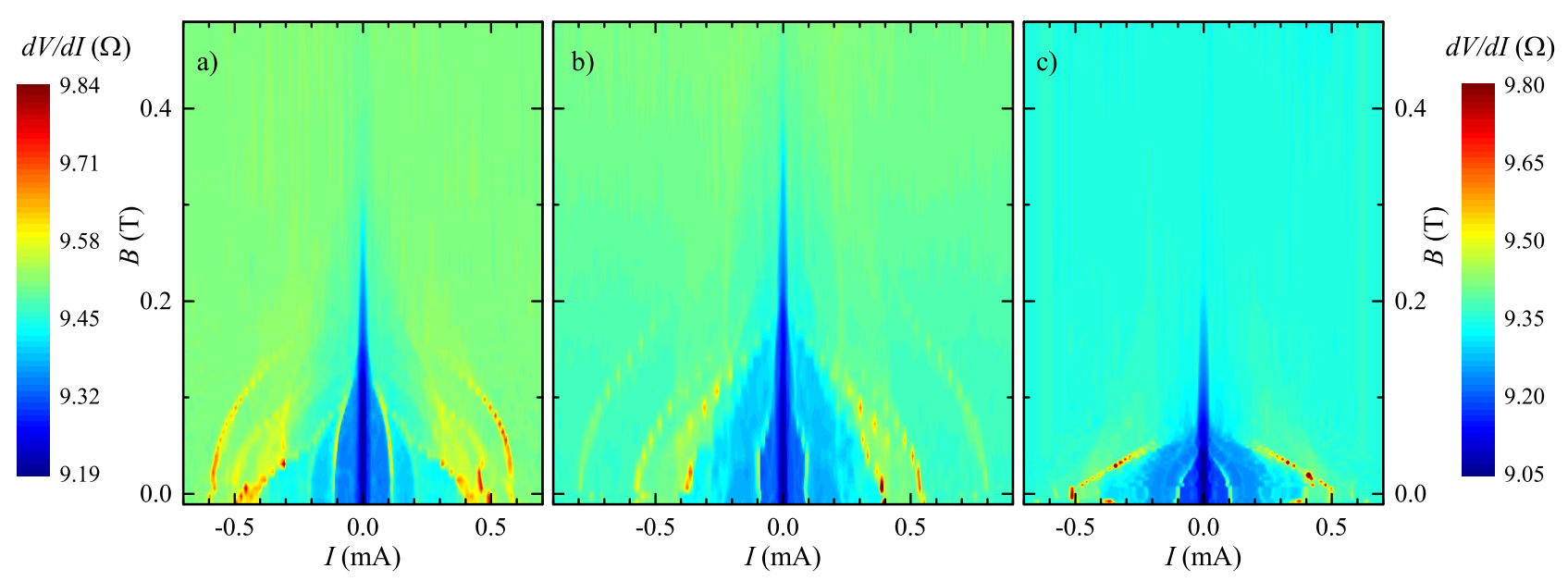

FIG. 5. (Color online) Evolution of $d V / d I(I)$ curves with magnetic field, which is applied along a, b and c WTe 2 crystal axes, respectively. Qualitative effect is similar: the level of $d V / d I(I)$ high-current saturation is constant; the zero-bias nonlinearity is suppressed; the positions of $d V / d I$ current-induced switchings are shifting to smaller currents. The effect is stronger in normal field, while there is no difference for two in-plane orientations. Color scale on the left reflects differential resistance levels in (a), color scale on the right refers to (b) and (c). The curves are obtained at $30 \mathrm{mK}$ for the transparent $\mathrm{Ni}^{-\mathrm{WTe}_{2}} \mathrm{junction}$ from Fig. 3 (a). The gradual evolution of switchings' positions also proves excellent reproducibility of these $d V / d I$ features.

ases, temperatures, or magnetic fields, see Figs. 3 (a), 4 (a), and 5, At zero bias, one can expect that spin polarization of some carriers at the $\mathrm{WTe}_{2}$ surface is aligned parallel to one in the ferromagnet due to the complicated spin texture of the topological Fermi arc surface state, see Fig. [1] This allows a direct transport channel even for spin-polarized carriers, which is reflected in low junction resistance at zero bias. When increasing the current through the surface state, spin-momentum locking [10, 11, 13, 14] produces preferable spin polarization. It suppresses transport due to the requirement on spin rotation in transport events, which is reflected as the overall $d V / d I$ increase for both signs of the current. This picture is consistent with the magnetic-field and temperature dependences of $d V / d I(I)$ : spin alignment at zero bias disappears when high magnetic field or temperature destroys spin textures of the topological surface state, so the zero-bias differential resistance is at

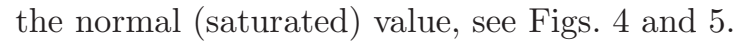

Similarly to the transparent metallic junctions [21, 22, the onset of the current-driven magnon excitations appears as $d V / d I$ peaks in Fig. 3. In low magnetic fields, the peaks positions are shifted [22] to lower currents, see Fig. 5, because an external field simplifies spin-wave excitation in the $\mathrm{WTe}_{2}$ surface state. We wish to emphasize, that the magnon excitation occurs in the $\mathrm{WTe}_{2}$ surface state, since transport properties of $\mathrm{Ni}$ layer and well compensated $\mathrm{WTe}_{2}$ bulk carriers [9] are invariant below $1 \mathrm{~K}$. Thus, our results can be regarded as direct manifestation of spin textures in $\mathrm{WTe}_{2}$ surface states in transport experiment.
As a conclusion, we experimentally investigate spinpolarized transport between a ferromagnetic Ni electrode and a surface of Weyl semimetal, realized in a thick $\mathrm{WTe}_{2}$ single crystal. For highly-transparent $\mathrm{Ni}-\mathrm{WTe}_{2}$ planar junctions, we observe non-Ohmic $d V / d I(I)$ behavior with an overall increase of differential resistance $d V / d I$ with current bias, which is accomplished by current-induced switchings. This behavior is inconsistent with trivial interface scattering, but it is well known for spin-polarized transport with magnon emission. Thus, we interpret the experimental results in terms of spin wave excitation in spin textures in the $\mathrm{WTe}_{2}$ topological surface states, which is supported by the obtained magnetic field and temperature $d V / d I(I)$ dependencies.

We wish to thank V.T. Dolgopolov and S.A. Tarasenko for fruitful discussions, and S.S Khasanov for X-ray sample characterization. We gratefully acknowledge financial support by the RFBR (project No. 16-02-00405) and RAS.

[1] M. Z. Hasan and C. L. Kane, Rev. Mod. Phys. 82, 3045 (2010).

[2] X.-L. Qi and S.-C. Zhang, Rev. Mod. Phys. 83, 1057 (2011).

[3] A. Bansil, H. Lin, and T. Das, Rev. Mod. Phys. 88, 021004 (2016).

[4] C.-K. Chiu, J. C. Teo, A. P. Schnyder, and S. Ryu, Rev. Mod. Phys. 88, 035005 (2016).

[5] As a recent review see N.P. Armitage, E.J. Mele, and A. Vishwanath, Rev. Mod. Phys. 90, 015001 (2018). 
[6] P. Li, Y. Wen, X. He1, Q. Zhang, C. Xia, Z.-M. Yu, S.A. Yang, Z. Zhu, H.N. Alshareef, X.-X. Zhang, Nature Comm. 8, 2150 (2017)

[7] A.A. Soluyanov, D. Gresch, Z. Wang, Q. Wu, M. Troyer, X. Dai, B.A. Bernevig, Nature 527, 495 (2015).

[8] M.N. Ali, J. Xiong, S. Flynn, J. Tao, Q.D. Gibson, L.M. Schoop, T. Liang, N. Haldolaarachchige, M. Hirschberger, N.P. Ong, and R.J. Cava, Nature (London) 514, 205 (2014).

[9] H.Y. Lv, W.J. Lu, D.F. Shao, Y. Liu, S.G. Tan, and Y.P. Sun, Europhys. Lett. 110, 37004 (2015).

[10] J. Jiang, F. Tang, X.C. Pan, H.M. Liu, X.H. Niu, Y.X. Wang, D.F. Xu, H.F. Yang, B.P. Xie, F.Q. Song, P. Dudin, T.K. Kim, M. Hoesch, P.K. Das, I. Vobornik, X.G. Wan, and D.L. Feng, Phys. Rev. Lett. 115, 166601 (2015).

[11] D. Rhodes, S. Das, Q.R. Zhang, B. Zeng, N.R. Pradhan, N. Kikugawa, E. Manousakis, and L. Balicas, Phys. Rev. B 92, 125152 (2015).

[12] Y.Wang, K.Wang, J. Reutt-Robey, J. Paglione, and M. S. Fuhrer, Phys. Rev. B 93, 121108 (2016).

[13] P.K. Das, D.D. Sante, I. Vobornik, J. Fujii, T. Okuda, E. Bruyer, A. Gyenis, B.E. Feldman, J. Tao, R. Ciancio, G. Rossi, M.N. Ali, S. Picozzi, A. Yadzani, G. Panaccione, and R.J. Cava, Nature Comm. 7, 10847 (2016).

[14] B. Feng, Y.-H. Chan, Y. Feng, R.-Y. Liu,1 M.-Y. Chou, K. Kuroda, K. Yaji, A. Harasawa, P. Moras, A. Barinov, W. Malaeb, C. Bareille, T. Kondo, S. Shin, F. Komori, T.-C. Chiang, Y. Shi, and I. Matsuda, Phys Rev B 94, 195134 (2016).

[15] Y. Sun, Y. Zhang, C. Felser, B. Yan, Phys. Rev. Lett. 117, 146403 (2016).

[16] B.Q. Lv, S. Muff, T. Qian, Z.D. Song, S.M. Nie, N. Xu, P. Richard, C.E. Matt, N.C. Plumb, L.X. Zhao, G.F. Chen, Z. Fang, X. Dai, J.H. Dil, J. Mesot, M. Shi, H.M. Weng, and H. Ding, Phys. Rev. Lett. 115, 217601 (2015).

[17] S.-Y. Xu, I. Belopolski, D.S. Sanchez, M. Neupane, G. Chang, K. Yaji, Z. Yuan, C. Zhang, K. Kuroda,
G. Bian, C. Guo, H. Lu, T.-R. Chang, N. Alidoust, H. Zheng, C.-C. Lee, S.-M. Huang, C.-H. Hsu, H.T. Jeng, A. Bansil, T. Neupert, F. Komori, T. Kondo, S. Shin, H. Lin, S. Jia, and M.Z. Hasan, Phys. Rev. Lett. 116, 096801 (2016).

[18] Y. Tserkovnyak, A. Brataas, G.E.W. Bauer, and B.I. Halperin, Rev. Mod. Phys. 77, 1375 (2005).

[19] J.C. Slonczewski, J. Magn. Magn. Mater. 159, L1-L7 (1996).

[20] D. MacNeill, G.M. Stiehl, M.H.D. Guimaraes, R.A. Buhrman, J. Park and D.C. Ralph, Nature Physics 13, 300 (2017).

[21] M. Tsoi, A. G. M. Jansen, J. Bass, W.-C. Chiang, M. Seck, V. Tsoi, and P. Wyder, Phys. Rev. Lett., 80, 4281 (1998).

[22] M. Tsoi, A. G. M. Jansen, J. Bass, W.-C. Chiang, V. Tsoi and P. Wyder, Nature, 406, 46, (2000).

[23] O. P. Balkashin, V. V. Fisun, I. K. Yanson, L. Yu. Triputen, A. Konovalenko, and V. Korenivski, Phys. Rev. B, 79, 092419 (2009)

[24] T. Balashov, A. F. Takács, 1 M. Däne, A. Ernst, P. Bruno, and W. Wulfhekel, Phys. Rev. B, 78, 174404 (2008)

[25] E. B. Borisenko, V. A. Berezin, N. N. Kolesnikov, V. K. Gartman, D. V. Matveev, O. F. Shakhlevich, Physics of the Solid State, 59, 1310, (2017).

[26] A. Sidorov, A.E. Petrova, A.N. Pinyagin, N.N. Kolesnikov, S.S. Khasanov, S.M. Stishov, JETP, 122, 1047, (2016).

[27] E.B. Myers, D.C. Ralph, J.A. Katine, R.N. Louie, R.A. Buhrman, Science, 285, 867 (1999).

[28] O. O. Shvetsov, A. Kononov, A. V. Timonina, N. N. Kolesnikov, E. V. Deviatov, JETP Letters, (2018) https://doi.org/10.1134/S0021364018120020

[29] A. Kononov, O. O. Shvetsov, S. V. Egorov, A. V. Timonina, N. N. Kolesnikov and E. V. Deviatov EPL, 122, 27004 (2018) doi: 10.1209/0295-5075/122/27004 